\title{
Leibniz algebras with absolute maximal Lie subalgebras
}

\author{
G. R. Biyogmam and C. Tcheka \\ Communicated by L. A. Kurdachenko
}

\begin{abstract}
A BStRACT. A Lie subalgebra of a given Leibniz algebra is said to be an absolute maximal Lie subalgebra if it has codimension one. In this paper, we study some properties of non-Lie Leibniz algebras containing absolute maximal Lie subalgebras. When the dimension and codimension of their Lie-center are greater than two, we refer to these Leibniz algebras as $s$-Leibniz algebras (strong Leibniz algebras). We provide a classification of nilpotent Leibniz $s$-algebras of dimension up to five.
\end{abstract}

\section{Introduction}

Leibniz algebras were introduced by Jean-Louis Loday (see [16]) as noncommutative versions of Lie algebras. Several authors (see $[2,3,5,6,11$, 12] for examples) have investigated whether the results on Lie algebras can be extended to Leibniz algebras. In [5] for instance, D. Barnes proved an analogue of Levi's decomposition for Leibniz algebras, which states that every finite dimensional Leibniz algebra $\mathfrak{g}$ over a field of characteristic zero can be written as a direct sum of its solvable radical and a semisimple Lie subalgebra. Note that in this theorem, the Lie algebra $\mathfrak{s}$ is not necessarily a maximal Lie subalgebra of $\mathfrak{g}$. In this paper, we examine non-Lie Leibniz algebras that can be written as semi-direct sum in which one summand is a maximal Lie subalgebra of codimension one. We will refer to these Lie subalgebras to as absolute maximal Lie subalgebras. Maximal subalgebras

2010 MSC: 17A32, 17B55, 18B99.

Key words and phrases: Leibniz algebras, $s$-Leibniz algebras, Lie-center. 
of codimension one in Lie algebras have been studied in $[1,17,18]$. We will particularly focus on the Leibniz algebras containing Lie subalgebras of codimension one, for which the Lie-center has dimension and codimension greater than two. We call them $s$-Leibniz algebras. We investigate their properties in section 3. This work opens the interesting debate as to evaluate how much properties does an $s$-Leibniz algebra inherit from its absolute maximal Lie subalgebra structure. For instance, we prove that an $s$-Leibniz algebra $\mathfrak{g}$ with absolute maximal Lie subalgebra $\mathfrak{s}$ is nilpotent and solvable if $\mathfrak{s}$ is a nilpotent ideal. Also, we provide certain conditions under which a Leibniz algebra is an $s$-Leibniz algebra. We also show that all absolute maximal Lie subalgebras of an $s$-Leibniz algebra meet at its Lie-center. In section 4 , we provide a classification of nilpotent $s$-Leibniz algebra of dimension up to five.

\section{Leibniz algebras}

We fix $\mathbb{K}$ as a ground field such that $\frac{1}{2} \in \mathbb{K}$. All vector spaces and tensor products are considered over $\mathbb{K}$. A (left) Leibniz algebra $[15,16]$ is a vector space $\mathfrak{g}$ equipped with a bilinear map $[-,-]: \mathfrak{g} \otimes \mathfrak{g} \rightarrow \mathfrak{g}$, usually called the Leibniz bracket of $\mathfrak{g}$, satisfying the Leibniz identity:

$$
[x,[y, z]]=[[x, y], z]+[y,[x, z]], \quad x, y, z \in \mathfrak{g} .
$$

Leibniz algebras form a semi-abelian category [7, 14], denoted by Leib, whose morphisms are linear maps that preserve the Leibniz bracket. The Leibniz identity above generalizes the Jacobi identity since $\mathfrak{g}$ becomes a Lie algebra when this bracket is antisymmetric. This defines the inclusion functor Lie $\hookrightarrow$ Leib. For a Leibniz algebra $\mathfrak{g}$, we denote by $\mathfrak{g}^{\text {ann }}$ the subspace of $\mathfrak{g}$ spanned by all elements of the form $[x, x], x \in \mathfrak{g}$. Given a Leibniz algebra $\mathfrak{g}$, it is clear that the quotient $\mathfrak{g}_{\text {Lie }}=\mathfrak{g} / \mathfrak{g}^{\text {ann }}$ is a Lie algebra. This defines a left adjoint functor to Lie $\hookrightarrow$ Leib, known as the Liezation functor $(-)_{\text {Lie }}:$ Leib $\rightarrow$ Lie, which assigns the Lie algebra $\mathfrak{g}_{\text {Lie }}$ to a given Leibniz algebra $\mathfrak{g}$.

A subalgebra $\mathfrak{h}$ of a Leibniz algebra $\mathfrak{g}$ is said to be left (resp. right) ideal of $\mathfrak{g}$ if $[h, q] \in \mathfrak{h}$ (resp. $[q, h] \in \mathfrak{h})$, for all $h \in \mathfrak{h}, q \in \mathfrak{g}$. If $\mathfrak{h}$ is both left and right ideal, then $\mathfrak{h}$ is called two-sided ideal of $\mathfrak{g}$. In this case $\mathfrak{g} / \mathfrak{h}$ naturally inherits a Leibniz algebra structure. The Lie-center of the Leibniz algebra $\mathfrak{g}$ is the two-sided ideal

$$
Z_{\text {Lie }}(\mathfrak{g})=\{z \in \mathfrak{g} \mid[g, z]+[z, g]=0 \text { for all } g \in \mathfrak{g}\}
$$


For a Leibniz algebra $\mathfrak{g}$ and two-sided ideals $\mathfrak{m}$ and $\mathfrak{n}$ of $\mathfrak{g}$, the Lie-centralizer of $\mathfrak{m}$ and $\mathfrak{n}$ over $\mathfrak{g}$ is

$$
C_{\mathfrak{g}}^{\mathrm{Lie}}(\mathfrak{m}, \mathfrak{n})=\{g \in \mathfrak{g} \mid[g, m]+[m, g] \in \mathfrak{n}, \text { for all } m \in \mathfrak{m}\}
$$

In particular, the Lie-centralizer of $\mathfrak{m}$ is defined by

$$
C_{\mathfrak{g}}^{\mathrm{Lie}}(\mathfrak{m}, 0)=\{g \in \mathfrak{g} /[g, m]+[m, g]=0, \text { for all } m \in \mathfrak{m}\}
$$

These notions of Lie-center and Lie-centralizer were studied in [4,6,8,9] as a result of approaching the relative theory of Leibniz algebras with respect to the Liezation functor. Note that $Z_{\text {Lie }}(\mathfrak{g})=\left\{x \in \mathfrak{g} \mid C_{\mathfrak{g}}^{\mathrm{Lie}}(\{x\}, 0)=\mathfrak{g}\right\}$. Also, $[x, y]_{\text {Lie }}=[x+y, x+y]-[x, x]-[y, y]$ for all $x, y \in \mathfrak{g}$. Consequently, $[\mathfrak{g}, \mathfrak{g}]_{\text {Lie }} \subseteq \mathfrak{g}^{a n n}$. Considering the set $T=\{t \in \mathfrak{g} \mid[t, t]=0\}$, it is clear that the sets $T$ and $\mathfrak{g}$ are equal iff $\mathfrak{g}$ is a Lie algebra.

\section{Leibniz algebras with absolute maximal Lie subalgebras}

In this section, we describe non-Lie Leibniz algebras containing absolute maximal Lie subalgebras. We discuss the cases where $\operatorname{dim}\left(Z_{\text {Lie }}(\mathfrak{g})\right)=n-1$ or $\operatorname{dim}\left(Z_{\text {Lie }}(\mathfrak{g})\right)=1$, and pay a particular attention to the non-Lie Leibniz algebras $\mathfrak{g}$ satisfying $1<\operatorname{dim}\left(Z_{\text {Lie }}(\mathfrak{g})\right)<n-1$.

Definition 3.1. Let $\mathfrak{g}$ be a Leibniz algebra. A subalgebra $\mathfrak{s}$ of $\mathfrak{g}$ is called absolute maximal Lie subalgebra if $\mathfrak{s}$ is a maximal Lie subalgebra of $\mathfrak{g}$ of codimension one.

Clearly, if $\mathfrak{g}$ is a Lie algebra, then all maximal subalgebras of codimension one are absolute maximal Lie subalgebras. Studies of maximal subalgebras of codimension one in Lie algebras can be found in $[1,17,18]$. For the remaining of the paper, we are interested in non-Lie Leibniz algebras.

Proposition 3.2. Let $\mathfrak{g}$ be a finite dimensional non-Lie Leibniz algebra and $\mathfrak{s}$ a maximal Lie subalgebra of $\mathfrak{g}$. Then $Z_{\text {Lie }}(\mathfrak{g})$ is a 2-sided ideal of $\mathfrak{s}$.

Proof. It is enough to show that $Z_{\text {Lie }}(\mathfrak{g}) \subseteq \mathfrak{s}$ since $Z_{\text {Lie }}(\mathfrak{g})$ is a 2-sided ideal of $\mathfrak{g}$. Indeed, let $g \in Z_{\text {Lie }}(\mathfrak{g})$ and assume that $g \notin \mathfrak{s}$. Then, $L:=$ $\operatorname{span}\{g, \mathfrak{s}\}=\mathfrak{g}$ since $\mathfrak{s}$ is a maximal subalgebra of $\mathfrak{g}$. Moreover $L$ is a Lie algebra because $[g, x]=-[x, g]$ for all $x \in \mathfrak{g}$ as $g \in Z_{\text {Lie }}(\mathfrak{g})$. This contradicts the fact that $\mathfrak{g}$ is a non-Lie Leibniz algebra. 
Lemma 3.3. Let $\mathfrak{g}$ be a $n$-dimensional non-Lie Leibniz algebra such that $\left(\mathfrak{g}-Z_{\text {Lie }}(\mathfrak{g})\right) \cap T \neq \varnothing$. The following is true:

a) $Z_{\text {Lie }}(\mathfrak{g})$ is not a maximal Lie subalgebra of $\mathfrak{g}$,

b) $\operatorname{dim}\left(Z_{\text {Lie }}(\mathfrak{g})\right)<n-1$,

c) If $T$ is a subalgebra of $\mathfrak{g}$ and $\left|\left(\mathfrak{g}-Z_{\text {Lie }}(\mathfrak{g})\right) \cap T\right|>k$, then $\operatorname{dim}\left(Z_{\text {Lie }}(\mathfrak{g})\right)<n-k$ for any $k<n$.

Proof. To prove a), let $a \notin Z_{\text {Lie }}(\mathfrak{g})$ and $a \in T$. By definition of $Z_{\text {Lie }}(\mathfrak{g})$, we have $[x, a]_{\text {Lie }}=0$ for all $x \in Z_{\text {Lie }}(\mathfrak{g})$. It follows that $\mathfrak{h}:=\operatorname{span}\left\{Z_{\text {Lie }}(\mathfrak{g}), a\right\}$ is a Lie subalgebra of $\mathfrak{g}$ strictly containing $Z_{\text {Lie }}(\mathfrak{g})$. Moreover, if $\operatorname{dim}\left(Z_{\text {Lie }}(\mathfrak{g})\right)=n-1$, then $\mathfrak{g}=\mathfrak{h}$ which contradicts the fact that $\mathfrak{g}$ is a non-Lie Leibniz algebra. This proves b).

To prove c), consider $k<l<n$ fixed, and let $a_{1} \ldots a_{l} \notin Z_{\text {Lie }}(\mathfrak{g})$ with $\left[a_{i}, a_{i}\right]=0$ for all $1 \leqslant i \leqslant l$. Clearly $\left[x, a_{i}\right]_{\text {Lie }}=0$ for all $x \in$ $Z_{\text {Lie }}(\mathfrak{g})$ and $1 \leqslant i \leqslant l$. Also, since $T$ is a subalgebra of $\mathfrak{g}$, we have $\left[a_{i}, a_{j}\right]_{\text {Lie }}=\left[a_{i}+a_{j}, a_{i}+a_{j}\right]-\left[a_{i}, a_{i}\right]-\left[a_{j}, a_{j}\right]=0$. It follows that $\mathfrak{h}:=$ $\operatorname{span}\left\{Z_{\text {Lie }}(\mathfrak{g}), a_{1}, \ldots, a_{l}\right\}$ is a Lie subalgebra of $\mathfrak{g}$. Since $\mathfrak{g}$ is a non-Lie Leibniz algebra of dimension $n$, it follows that $\operatorname{dim}\left(Z_{\text {Lie }}(\mathfrak{g})\right)+l<n$, and thus $\operatorname{dim}\left(Z_{\text {Lie }}(\mathfrak{g})\right)<n-l<n-k$.

Proposition 3.4. Let $\mathfrak{g}$ be a n-dimensional non-Lie Leibniz algebra, and $\mathfrak{s}$ a Lie subalgebra of $\mathfrak{g}$ such that $(\mathfrak{g}-\mathfrak{s}) \cap T \neq \varnothing$. If $T$ is a subalgebra of $\mathfrak{g}$, then $\mathfrak{s}$ is not a maximal Lie subalgebra of $\mathfrak{g}$, and $\operatorname{dim}(\mathfrak{s})<n-1$.

Proof. Let $a \notin \mathfrak{s}$ and $a \in T$, i.e. $[a, a]=0$. Since $T$ is a subalgebra of $\mathfrak{g}$, we have $[x, a]_{\text {Lie }}=[x+a, x+a]-[x, x]-[a, a]=0$ for all $x \in \mathfrak{s}$. It follows that $\mathfrak{h}:=\operatorname{span}\{\mathfrak{s}, a\}$ is a Lie subalgebra of $\mathfrak{g}$ strictly containing $\mathfrak{s}$. Moreover, if $\operatorname{dim}(\mathfrak{s})=n-1$, then $\mathfrak{g}=\mathfrak{h}$ which contradicts the fact that $\mathfrak{g}$ is a non-Lie Leibniz algebra.

Corollary 3.5. Let $\mathfrak{g}$ be a finite dimensional non-Lie Leibniz algebra such that $\operatorname{dim}\left(\frac{\mathfrak{g}}{Z_{\text {Lie }}(\mathfrak{g})}\right)=1$. Then $Z_{\text {Lie }}(\mathfrak{g})$ is the unique absolute maximal Lie subalgebra of $\mathfrak{g}$.

Proof. Since $\operatorname{dim}\left(Z_{\text {Lie }}(\mathfrak{g})\right)=\operatorname{dim}(\mathfrak{g})-1$, one easily verifies that $Z_{\text {Lie }}(\mathfrak{g})$ is a maximal Lie subalgebra of $\mathfrak{g}$. Now by Proposition $3.2, Z_{\text {Lie }}(\mathfrak{g})$ is contained in all maximal Lie subalgebra of $\mathfrak{g}$. The result follows by the maximality of $Z_{\text {Lie }}(\mathfrak{g})$.

Example 3.6. Consider the Leibniz algebra $\mathfrak{g}$ spanned by $\left\{a_{1}, a_{2}, a_{3}\right.$, $\left.a_{4}, a_{5}\right\}$ with nonzero bracket $\left[a_{1}, a_{2}\right]=a_{5},\left[a_{2}, a_{1}\right]=-a_{5},\left[a_{3}, a_{4}\right]=$ 
$a_{5},\left[a_{4}, a_{3}\right]=-a_{5},\left[a_{4}, a_{4}\right]=a_{5}$. Then one can verify that $Z_{\text {Lie }}(\mathfrak{g})=$ $\operatorname{span}\left\{a_{1}, a_{2}, a_{3}, a_{5}\right\}$ is the unique maximal Lie subalgebra of $\mathfrak{g}$.

Proposition 3.7. Let $\mathfrak{g}$ be a n-dimensional non-Lie left central Leibniz algebra with one dimensional Lie-center. Then $\mathfrak{t}=\operatorname{span}\{t \in \mathfrak{g} \mid[t, t]=0\}$ is an absolute maximal Lie subalgebra of $\mathfrak{g}$.

Proof. Since $\mathfrak{g}$ is left central, then for all $t \in \mathfrak{g},[t, t] \in Z_{\text {Lie }}(\mathfrak{g})$. It follows that $\frac{\mathfrak{g}}{Z_{\text {Lie }}(\mathfrak{g})}=\operatorname{span}\left\{t+Z_{\text {Lie }}(\mathfrak{g}),[t, t]=0\right\}$. Moreover, $\mathfrak{g}^{a n n}=Z_{\text {Lie }}(\mathfrak{g})$, so the map $\mathfrak{g} \rightarrow Z_{\text {Lie }}(\mathfrak{g})$ defined by $t \mapsto[t, t]$ induces an isomorphism $\frac{\mathfrak{g}}{\mathfrak{t}} \cong Z_{\text {Lie }}(\mathfrak{g})$. Therefore the set $\mathfrak{t}=\operatorname{span}\{t \in \mathfrak{g} \mid[t, t]=0\}$ is an absolute maximal Lie subalgebra of $\mathfrak{g}$.

Proposition 3.8. Let $\mathfrak{g}$ be a n-dimensional nilpotent non-Lie Leibniz algebra with $(n-1)$-dimensional derived subalgebra. Then $\operatorname{dim}\left(Z_{\text {Lie }}(\mathfrak{g})\right)=1$ and $[\mathfrak{g}, \mathfrak{g}]$ is the unique absolute maximal Lie subalgebra of $\mathfrak{g}$.

Proof. By [2, Lemma 1], $\mathfrak{g}$ is generated by $\left\{x_{1}, x_{2}, x_{3}, \ldots, x_{n}\right\}$ satisfying the conditions $\left[x_{i}, x_{1}\right]=x_{i+1}$ for $1 \leqslant i \leqslant n-1,\left[x_{i}, x_{j}\right]=0$ for $j \geqslant 2$. It is easily verified that $Z_{\text {Lie }}(\mathfrak{g})=\operatorname{span}\left\{x_{n}\right\}$ and $[\mathfrak{g}, \mathfrak{g}]=\operatorname{span}\left\{x_{2}, x_{3}, \ldots, x_{n}\right\}$ is the only absolute maximal Lie subalgebra of $\mathfrak{g}$.

The following definition introduces a subclass of Leibniz algebras called strong Leibniz algebras ( $s$-Leibniz algebras).

Definition 3.9. A n-dimensional non-Lie Leibniz algebra $\mathfrak{g}$ is said to be an s-Leibniz algebra if:

$(\mathrm{S} 1) \operatorname{dim}\left(Z_{\text {Lie }}(\mathfrak{g})\right) \geqslant 2$ and $\operatorname{codim}\left(Z_{\text {Lie }}(\mathfrak{g})\right) \geqslant 2$;

(S2) $\mathfrak{g}$ contains an absolute maximal Lie subalgebra.

Remark 3.10. Leibniz algebras of Corollary 3.5, Proposition 3.7 and Proposition 3.8 do not satisfy the condition (S1). So they are not $s$-Leibniz algebras.

Example 3.11. Consider the Leibniz algebra $\mathfrak{g}$ spanned by $\{a, b, c, d, e\}$ with nonzero bracket $[a, d]=e,[b, c]=e,[b, d]=e,[c, b]=-e,[c, c]=e$, $[d, a]=-e$ and $[d, b]=e$. Then one can verify that $Z_{\mathrm{Lie}}(\mathfrak{g})=\operatorname{span}\{a, e\}$, and $\mathfrak{g}$ contains no absolute maximal Lie subalgebra, and so $\mathfrak{g}$ is not an $s$-Leibniz algebra.

Example 3.12. Consider the Leibniz algebra $\mathfrak{g}$ spanned by $\left\{a_{1}, a_{2}, a_{3}\right.$, $\left.a_{4}, a_{5}\right\}$ with nonzero bracket $\left[a_{1}, a_{1}\right]=a_{4},\left[a_{1}, a_{2}\right]=a_{3},\left[a_{2}, a_{1}\right]=-a_{3}$, $\left[a_{2}, a_{3}\right]=a_{5},\left[a_{3}, a_{2}\right]=-a_{5},\left[a_{1}, a_{4}\right]=a_{5}$. Then one can verify that $Z_{\text {Lie }}(\mathfrak{g})=\operatorname{span}\left\{a_{2}, a_{3}, a_{5}\right\}$, and $\mathfrak{s}=\operatorname{span}\left\{a_{2}, a_{3}, a_{4}, a_{5}\right\}$ is an absolute maximal Lie subalgebra of $\mathfrak{g}$. So $\mathfrak{g}$ is an $s$-Leibniz algebra. 
Remark 3.13. Let $\mathfrak{g}$ be a finite dimensional $s$-Leibniz algebra with absolute maximal Lie subalgebra $\mathfrak{s}$. By Proposition $3.2, Z_{\text {Lie }}(\mathfrak{g})$ is a 2sided ideal of $\mathfrak{s}$.

Lemma 3.14. Let $\mathfrak{g}$ be a n-dimensional s-Leibniz algebra with a absolute maximal Lie subalgebra $\mathfrak{s}$. If $\mathfrak{s}$ is a two-sided ideal of $\mathfrak{g}$, then $[\mathfrak{g}, \mathfrak{g}] \subseteq \mathfrak{s}$.

Proof. Let $\langle x\rangle$ be the complement of $\mathfrak{s}$ in $\mathfrak{g}$. Then $[a, x],[x, a],[a, b] \in \mathfrak{s}$ for all $a, b \in \mathfrak{s}$. It remains to show that $[x, x] \in \mathfrak{s}$. Indeed assume that $[x, x]=\alpha x+h$ for some $\alpha \in \mathbb{R}$ and $h \in \mathfrak{s}$. Then by linearity and using the Leibniz identity, we have $\alpha[x, x]+[h, x]=[\alpha x+h, x]=[[x, x], x]=0 \in \mathfrak{s}$. Since $[h, x] \in \mathfrak{s}$, it follows that either $\alpha=0$ in which case $[x, x]=h$ or $[x, x]=0$.

Corollary 3.15. Let $\mathfrak{g}$ be a finite dimensional $s$-Leibniz algebra with absolute maximal Lie subalgebra $\mathfrak{s}$. If $\mathfrak{s}$ is a nilpotent two-sided ideal of $\mathfrak{g}$, then $\mathfrak{g}$ is solvable and nilpotent.

Proof. Using Lemma 3.14, one can easily verify that if $\mathfrak{s}$ is nilpotent, then $[\mathfrak{g}, \mathfrak{g}]$ is nilpotent, and thus $\mathfrak{g}$ is nilpotent. Moreover, $\mathfrak{g}$ is solvable by [13, Corollary 6].

Proposition 3.16. Let $\mathfrak{g}$ be s-Leibniz algebra and let $\mathfrak{S}$ be the set of all absolute maximal Lie subalgebras of $\mathfrak{g}$. If $|\mathfrak{S}| \neq 1$, then $Z_{\text {Lie }}(\mathfrak{g})=\bigcap_{\mathfrak{s} \in \mathfrak{S}} \mathfrak{s}$.

Proof. By Proposition 3.13, $Z_{\text {Lie }}(\mathfrak{g})$ is contained in every absolute maximal

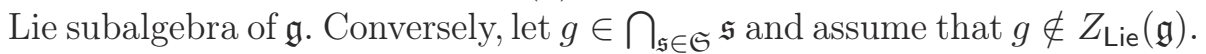

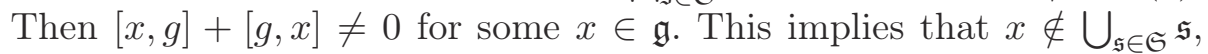
otherwise, $x, g \in \mathfrak{s}_{0}$ for some Lie subalgebra of $\mathfrak{g}$, which contradicts $[x, g]+[g, x] \neq 0$. So we have $\operatorname{span}\{x, \mathfrak{s}\}=\mathfrak{g}$ for all $\mathfrak{s} \in \mathfrak{S}$, and thus $|\mathfrak{S}|=1$. A contradiction.

Recall the following Levi's Theorem proven by D. Barnes for Leibniz algebras:

Theorem 3.17. [5] Every finite-dimensional Leibniz algebra $\mathfrak{g}$ over a field of characteristic 0 can be written as $\mathfrak{g}=\mathfrak{s} \oplus \operatorname{Rad}(\mathfrak{g})$, where $\mathfrak{s}$ is a semisimple Lie subalgebra of $\mathfrak{g}$, and $\operatorname{Rad}(\mathfrak{g})$ is the solvable radical of $\mathfrak{g}$.

Proposition 3.18. Let $\mathfrak{g}$ be a Leibniz algebra satisfying (S1), and $\mathfrak{h}$ an absolute maximal subalgebra of $\mathfrak{g}$ containing $Z_{\text {Lie }}(\mathfrak{g})$, and such that $\mathfrak{h} / Z_{\text {Lie }}(\mathfrak{g})$ is a semisimple Lie subalgebra of $\mathfrak{g} / Z_{\text {Lie }}(\mathfrak{g})$. Then $\mathfrak{g}$ is an $s$-Leibniz algebra with absolute maximal Lie subalgebra $\mathfrak{h}$. 
Proof. By Theorem 3.17, $\mathfrak{h}=\mathfrak{s} \oplus \operatorname{Rad}(\mathfrak{h})$ for some semisimple Lie subalgebra $\mathfrak{s}$ of $\mathfrak{h}$. But since $\mathfrak{h} / Z_{\text {Lie }}(\mathfrak{g})$ is a semisimple Lie subalgebra of $\mathfrak{g} / Z_{\text {Lie }}(\mathfrak{g})$, it follows that $\mathfrak{h}=Z_{\text {Lie }}(\mathfrak{g})+\mathfrak{s}$, which is clearly a Lie-subalgebra of $\mathfrak{g}$. Therefore $\mathfrak{h}$ is an absolute maximal Lie subalgebra of $\mathfrak{g}$.

Corollary 3.19. Let $\mathfrak{g}$ be a Leibniz algebra satisfying (S1), and such that $\mathfrak{g} / Z_{\text {Lie }}(\mathfrak{g})$ contains an absolute maximal semisimple subalgebra. Then $\mathfrak{g}$ is an s-Leibniz algebra.

Proof. Let $\mathfrak{h} / Z_{\text {Lie }}(\mathfrak{g})$ is an absolute maximal semisimple subalgebra of $\mathfrak{g} / Z_{\text {Lie }}(\mathfrak{g})$. Then by the proof of Proposition $3.18, \mathfrak{h}$ is a Lie subalgebra of $\mathfrak{g}$. It is easy to show that $\mathfrak{h}$ is an absolute maximal subalgebra of $\mathfrak{g}$.

\section{Classification of all nilpotent $s$-Leibniz algebras dimension at most 5}

In this section, we use the classification of four and five dimensional nilpotent Leibniz Algebras provided in [10] to determine all nilpotent $s$ Leibniz algebras of dimension four and five. This consists for each Leibniz algebra to find the Lie-center and the absolute maximal Lie subalgebra (if it exists), and verify satisfaction of the condition (S1) on the Lie-center. As pointed out in [10], these Leibniz algebras are non-split, i.e. they cannot be written as a direct sum of two nontrivial ideals. Split Leibniz algebras can be obtained from non split ones.

Theorem 4.1. Every four dimensional non-split nilpotent s-Leibniz algebra is isomorphic to a Leibniz algebra spanned by $\left\{x_{1}, x_{2}, x_{3}, x_{4}\right\}$ where the nonzero products are given by following:

A12: $\left[x_{1}, x_{1}\right]=x_{4},\left[x_{1}, x_{2}\right]=x_{3},\left[x_{2}, x_{1}\right]=-x_{3}+x_{4},\left[x_{1}, x_{3}\right]=x_{4}=-\left[x_{3}, x_{1}\right]$. A13: $\left[x_{1}, x_{2}\right]=x_{3},\left[x_{2}, x_{1}\right]=-x_{3}+x_{4},\left[x_{2}, x_{2}\right]=x_{4},\left[x_{1}, x_{3}\right]=x_{4}=-\left[x_{3}, x_{1}\right]$.

A14: $\left[x_{1}, x_{1}\right]=x_{3},\left[x_{1}, x_{2}\right]=x_{4}$.

A15: $\left[x_{1}, x_{1}\right]=x_{3},\left[x_{2}, x_{1}\right]=x_{4}$.

A16: $\left[x_{1}, x_{2}\right]=x_{4},\left[x_{2}, x_{1}\right]=x_{3},\left[x_{2}, x_{2}\right]=-x_{3}$.

$\mathrm{A} 17:\left[x_{1}, x_{1}\right]=x_{3},\left[x_{1}, x_{2}\right]=x_{4},\left[x_{2}, x_{1}\right]=\alpha x_{4}, \alpha \in C /\{-1,0\}$

Proof. The result is obtained by checking each Leibniz algebra of Theorem 3.2.3 in [10]. We kept the same numbering of these Leibniz algebras as in [10] for the convenience of the reader.

The basis of the corresponding Lie-centers and absolute maximal Lie subalgebras are given in the table below: 


\begin{tabular}{|l|l|l|}
\hline \multicolumn{2}{|c|}{ 4-dimensional non-split nilpotent $s$-Leibniz algebras } \\
\hline$s$-Leibniz algebra $\mathfrak{g}$ & Lie-center $Z_{\text {Lie }}(\mathfrak{g})$ & $\begin{array}{l}\text { Absolute maximal Lie } \\
\text { subalgebra } \mathfrak{s}\end{array}$ \\
\hline A12, A14, A15, A17 & $\left\{x_{3}, x_{4}\right\}$ & $\left\{x_{2}, x_{3}, x_{4}\right\}$ \\
\hline \hline A13, A16 & $\left\{x_{3}, x_{4}\right\}$ & $\left\{x_{1}, x_{3}, x_{4}\right\}$ \\
\hline
\end{tabular}

Theorem 4.2. Every five dimensional non-split nilpotent s-Leibniz algebra is isomorphic to a Leibniz algebra spanned by $\left\{x_{1}, x_{2}, x_{3}, x_{4}\right\}$ where the nonzero products are given by following:

B1: $\left[x_{1}, x_{2}\right]=x_{3},\left[x_{2}, x_{1}\right]=-x_{3}+x_{5},\left[x_{1}, x_{3}\right]=x_{4}=-\left[x_{3}, x_{1}\right] .$.

B3: $\left[x_{1}, x_{2}\right]=x_{3},\left[x_{2}, x_{1}\right]=-x_{3}+x_{5},\left[x_{2}, x_{2}\right]=x_{5},\left[x_{1}, x_{3}\right]=x_{4}=$ $-\left[x_{3}, x_{1}\right]$.

$\mathrm{C} 2:\left[x_{1}, x_{2}\right]=x_{3},\left[x_{2}, x_{1}\right]=-x_{3}+x_{4},\left[x_{2}, x_{2}\right]=x_{5},\left[x_{1}, x_{3}\right]=x_{5}=$ $-\left[x_{3}, x_{1}\right]$.

C4: $\left[x_{1}, x_{2}\right]=x_{3},\left[x_{2}, x_{1}\right]=-x_{3}+x_{5},\left[x_{2}, x_{2}\right]=x_{4},\left[x_{1}, x_{3}\right]=x_{4}=$ $-\left[x_{3}, x_{1}\right]$.

C8: $\left[x_{1}, x_{1}\right]=x_{4},\left[x_{1}, x_{2}\right]=x_{3},\left[x_{2}, x_{1}\right]=-x_{3}+x_{5},\left[x_{1}, x_{3}\right]=x_{4}=$ $-\left[x_{3}, x_{1}\right]$.

D1: $\left[x_{1}, x_{1}\right]=x_{3},\left[x_{2}, x_{1}\right]=-x_{4},\left[x_{1}, x_{3}\right]=x_{5}$.

D8: $\left[x_{1}, x_{1}\right]=x_{3},\left[x_{1}, x_{2}\right]=x_{4},\left[x_{2}, x_{1}\right]=\alpha x_{4},\left[x_{1}, x_{3}\right]=x_{5}, \alpha \in$ $\mathbb{C} /\{-1\}$.

D14: $\left[x_{1}, x_{1}\right]=x_{4},\left[x_{1}, x_{2}\right]=x_{3},\left[x_{1}, x_{3}\right]=x_{5}$.

D15: $\left[x_{1}, x_{1}\right]=x_{4},\left[x_{1}, x_{2}\right]=x_{3},\left[x_{2}, x_{1}\right]=x_{5},\left[x_{1}, x_{3}\right]=x_{5}$

D18: $\left[x_{1}, x_{1}\right]=x_{4},\left[x_{1}, x_{2}\right]=x_{3},\left[x_{2}, x_{1}\right]=x_{4},\left[x_{1}, x_{3}\right]=x_{5}$.

E1: $\left[x_{1}, x_{2}\right]=-x_{3}+x_{4},\left[x_{2}, x_{1}\right]=x_{3},\left[x_{2}, x_{3}\right]=x_{5},\left[x_{3}, x_{2}\right]=-x_{5}$, $\left[x_{1}, x_{4}\right]=x_{5}$.

E10: $\left[x_{1}, x_{1}\right]=x_{4},\left[x_{1}, x_{2}\right]=x_{3},\left[x_{2}, x_{1}\right]=-x_{3},\left[x_{2}, x_{3}\right]=x_{5},\left[x_{3}, x_{2}\right]=$ $-x_{5},\left[x_{1}, x_{4}\right]=x_{5}$.

F1: $\left[x_{1}, x_{2}\right]=x_{3},\left[x_{2}, x_{1}\right]=-x_{3}+x_{5},\left[x_{1}, x_{3}\right]=x_{4},\left[x_{3}, x_{1}\right]=-x_{4}$, $\left[x_{2}, x_{3}\right]=x_{5},\left[x_{3}, x_{2}\right]=-x_{5}$.

F3: $\left[x_{1}, x_{2}\right]=x_{3},\left[x_{2}, x_{1}\right]=-x_{3}+x_{5},\left[x_{2}, x_{2}\right]=x_{5},\left[x_{1}, x_{3}\right]=x_{4}$, $\left[x_{3}, x_{1}\right]=-x_{4},\left[x_{2}, x_{3}\right]=x_{5},\left[x_{3}, x_{2}\right]=-x_{5}$.

G1: $\left[x_{1}, x_{2}\right]=x_{3},\left[x_{2}, x_{1}\right]=-x_{3}+x_{4},\left[x_{2}, x_{2}\right]=\alpha x_{5},\left[x_{1}, x_{3}\right]=x_{4}$, $\left[x_{3}, x_{1}\right]=-x_{4},\left[x_{2}, x_{3}\right]=x_{5},\left[x_{3}, x_{2}\right]=-x_{5}, \alpha \in \mathbb{C} /\{-1\}$.

G3: $\left[x_{1}, x_{1}\right]=\alpha x_{5},\left[x_{1}, x_{2}\right]=x_{3},\left[x_{2}, x_{1}\right]=-x_{3}+x_{4}+x_{5},\left[x_{1}, x_{3}\right]=x_{4}$, $\left[x_{3}, x_{1}\right]=-x_{4},\left[x_{2}, x_{3}\right]=x_{5},\left[x_{3}, x_{2}\right]=-x_{5}, \alpha \in \mathbb{C} /\{-1\}$.

$\mathrm{H} 1:\left[x_{1}, x_{2}\right]=x_{3},\left[x_{2}, x_{1}\right]=-x_{3}+x_{5},\left[x_{1}, x_{3}\right]=x_{4},\left[x_{3}, x_{1}\right]=-x_{4}$, $\left[x_{1}, x_{4}\right]=x_{5},\left[x_{4}, x_{1}\right]=-x_{5}$.

H3: $\left[x_{1}, x_{2}\right]=x_{3},\left[x_{2}, x_{1}\right]=-x_{3}+x_{5},\left[x_{2}, x_{2}\right]=x_{5},\left[x_{1}, x_{3}\right]=x_{4}$, $\left[x_{3}, x_{1}\right]=-x_{4},\left[x_{1}, x_{4}\right]=x_{5},\left[x_{4}, x_{1}\right]=-x_{5}$. 
H5: $\left[x_{1}, x_{2}\right]=x_{3},\left[x_{2}, x_{1}\right]=-x_{3}+x_{5},\left[x_{2}, x_{2}\right]=\alpha x_{5},\left[x_{1}, x_{3}\right]=x_{4}$, $\left[x_{2}, x_{3}\right]=x_{5},\left[x_{3}, x_{2}\right]=-x_{5},\left[x_{3}, x_{1}\right]=-x_{4},\left[x_{1}, x_{4}\right]=x_{5},\left[x_{4}, x_{1}\right]=$ $-x_{5}, \alpha \in \mathbb{C}$.

I6: $\left[x_{1}, x_{2}\right]=x_{4},\left[x_{2}, x_{1}\right]=-x_{4},\left[x_{1}, x_{3}\right]=x_{5},\left[x_{1}, x_{4}\right]=x_{5},\left[x_{4}, x_{1}\right]=$ $-x_{5}$.

I8: $\left[x_{1}, x_{2}\right]=x_{4},\left[x_{2}, x_{1}\right]=-x_{4},\left[x_{2}, x_{3}\right]=x_{5},\left[x_{1}, x_{4}\right]=x_{5},\left[x_{4}, x_{1}\right]=$ $-x_{5}$.

I13: $\left[x_{1}, x_{2}\right]=x_{4},\left[x_{2}, x_{1}\right]=-x_{4}+x_{5},\left[x_{2}, x_{3}\right]=-x_{5},\left[x_{3}, x_{2}\right]=x_{5}$, $\left[x_{1}, x_{4}\right]=x_{5},\left[x_{4}, x_{1}\right]=-x_{5}$.

I14: $\left[x_{1}, x_{2}\right]=x_{4},\left[x_{2}, x_{1}\right]=-x_{4},\left[x_{2}, x_{3}\right]=\alpha x_{5},\left[x_{3}, x_{2}\right]=x_{5},\left[x_{1}, x_{4}\right]=$ $x_{5},\left[x_{4}, x_{1}\right]=-x_{5}, \alpha \in \mathbb{C}$.

I18: $\left[x_{1}, x_{2}\right]=x_{4},\left[x_{2}, x_{1}\right]=-x_{4}+x_{5},\left[x_{2}, x_{2}\right]=x_{5},\left[x_{2}, x_{3}\right]=-x_{5}$, $\left[x_{3}, x_{2}\right]=x_{5},\left[x_{1}, x_{4}\right]=x_{5},\left[x_{4}, x_{1}\right]=-x_{5}$.

J8: $\left[x_{1}, x_{1}\right]=x_{4},\left[x_{2}, x_{3}\right]=\alpha x_{5},\left[x_{3}, x_{2}\right]=x_{5},\left[x_{1}, x_{4}\right]=x_{5}$.

$\mathrm{J} 10:\left[x_{1}, x_{2}\right]=x_{4},\left[x_{2}, x_{3}\right]=\alpha x_{5},\left[x_{3}, x_{2}\right]=x_{5},\left[x_{1}, x_{4}\right]=x_{5}$.

$\mathrm{K} 2:\left[x_{1}, x_{2}\right]=x_{4},\left[x_{2}, x_{1}\right]=-x_{4},\left[x_{3}, x_{1}\right]=x_{5}$.

$\mathrm{K} 4:\left[x_{1}, x_{2}\right]=x_{4},\left[x_{2}, x_{1}\right]=-x_{4},\left[x_{1}, x_{3}\right]=x_{5},\left[x_{3}, x_{1}\right]=\alpha x_{5}, \alpha \in$ $\mathbb{C} /\{-1\}$.

K5: $\left[x_{1}, x_{2}\right]=x_{4},\left[x_{2}, x_{1}\right]=-x 4+x_{5},\left[x_{1}, x_{3}\right]=x_{5},\left[x_{3}, x_{1}\right]=-x_{5}$.

K6: $\left[x_{1}, x_{2}\right]=x_{4},\left[x_{2}, x_{1}\right]=-x_{4},\left[x_{1}, x_{3}\right]=x_{5},\left[x_{3}, x_{1}\right]=-\alpha x_{5}$, $\left[x_{3}, x_{3}\right]=x_{5}, \alpha \in \mathbb{C} /\{-1\}$.

$\mathrm{K} 9:\left[x_{1}, x_{2}\right]=x_{4},\left[x_{2}, x_{1}\right]=-x_{4},\left[x_{1}, x_{3}\right]=x_{5},\left[x_{3}, x_{2}\right]=x_{5}$.

$\mathrm{K} 12:\left[x_{1}, x_{2}\right]=x_{4},\left[x_{2}, x_{1}\right]=-x_{4}+x_{5},\left[x_{1}, x_{3}\right]=x_{5},\left[x_{3}, x_{1}\right]=-x_{5}$, $\left[x_{2}, x_{3}\right]=x_{5},\left[x_{3}, x_{2}\right]=-x_{5}$.

L14: $\left[x_{1}, x_{2}\right]=x_{4},\left[x_{2}, x_{3}\right]=x_{5}$.

L18: $\left[x_{1}, x_{2}\right]=\alpha x_{4},\left[x_{2}, x_{1}\right]=x_{4},\left[x_{2}, x_{3}\right]=x_{5}, \alpha \in \mathbb{C} /\{-1\}$.

L35: $\left[x_{1}, x_{2}\right]=x_{5},\left[x_{2}, x_{2}\right]=x_{4},\left[x_{2}, x_{3}\right]=x_{5}$.

L36: $\left[x_{1}, x_{2}\right]=x_{4},\left[x_{2}, x_{1}\right]=-x_{4},\left[x_{2}, x_{2}\right]=x_{4},\left[x_{2}, x_{3}\right]=x_{5}$.

L42: $\left[x_{2}, x_{1}\right]=x_{4},\left[x_{1}, x_{3}\right]=x_{5},\left[x_{2}, x_{3}\right]=-x_{5},\left[x_{3}, x_{2}\right]=x_{5}$.

L44: $\left[x_{1}, x_{2}\right]=x_{4},\left[x_{2}, x_{1}\right]=\alpha x_{4},\left[x_{2}, x_{3}\right]=\beta x_{5},\left[x_{3}, x_{2}\right]=x_{5}, \alpha, \beta \in$ $\mathbb{C} /\{-1\}$.

L45: $\left[x_{1}, x_{1}\right]=x_{5},\left[x_{1}, x_{2}\right]=x_{4},\left[x_{2}, x_{1}\right]=\alpha x_{4},\left[x_{2}, x_{3}\right]=\beta x_{5},\left[x_{3}, x_{2}\right]=$ $x_{5}, \alpha \in \mathbb{C} /\{-1\}, \beta \in \mathbb{C}$.

L46: $\left[x_{1}, x_{2}\right]=x_{4},\left[x_{2}, x_{1}\right]=\alpha x_{4}+x_{5},\left[x_{2}, x_{3}\right]=\alpha x_{5},\left[x_{3}, x_{2}\right]=x_{5}$, $\alpha \in \mathbb{C} /\{-1\}$.

L50: $\left[x_{1}, x_{2}\right]=x_{4},\left[x_{2}, x_{1}\right]=\alpha x_{4},\left[x_{2}, x_{2}\right]=x_{5},\left[x_{2}, x_{3}\right]=x_{5},\left[x_{3}, x_{2}\right]=$ $-x_{5}, \alpha \in \mathbb{C} /\{-1\}$.

L55: $\left[x_{1}, x_{1}\right]=x_{4},\left[x_{2}, x_{1}\right]=x_{4},\left[x_{1}, x_{3}\right]=\alpha x_{5},\left[x_{2}, x_{3}\right]=-x_{5},\left[x_{3}, x 2\right]=$ $x_{5}, \alpha \in \mathbb{C} /\{0\}$.

L56: $\left[x_{1}, x_{1}\right]=x_{4},\left[x_{2}, x_{1}\right]=x_{4}+x_{5},\left[x_{2}, x_{3}\right]=x_{5},\left[x_{3}, x_{2}\right]=-x_{5}$. 
L58: $\left[x_{1}, x_{1}\right]=x_{4},\left[x_{1}, x_{2}\right]=x 4,\left[x_{2}, x_{1}\right]=-x_{4},\left[x_{1}, x_{3}\right]=\beta x_{5},\left[x_{2}, x_{3}\right]=$ $-x_{5},\left[x_{3}, x_{2}\right]=x_{5}, \beta \in \mathbb{C} /\{0\}$.

L60: $\left[x_{1}, x_{1}\right]=x_{4},\left[x_{1}, x_{2}\right]=x_{4}+x_{5},\left[x_{2}, x_{1}\right]=\alpha x_{4},\left[x_{2}, x_{3}\right]=-x_{5}$, $\left[x_{3}, x_{2}\right]=x_{5}, \alpha \in \mathbb{C}$.

L61: $\left[x_{1}, x_{1}\right]=x_{4},\left[x_{1}, x_{2}\right]=x_{4}+x_{5},\left[x_{2}, x_{1}\right]=x_{4},\left[x_{1}, x_{3}\right]=\alpha x_{5}$, $\left[x_{2}, x_{3}\right]=-x_{5},\left[x_{3}, x_{2}\right]=x_{5}, \alpha \in \mathbb{C} /\{-1,0\}$.

L63: $\left[x_{1}, x_{1}\right]=x_{4},\left[x_{1}, x_{2}\right]=x_{4}+x_{5},\left[x_{2}, x_{1}\right]=\alpha x_{4},\left[x_{1}, x_{3}\right]=\beta x_{5}$, $\left[x_{2}, x_{3}\right]=-x_{5},\left[x_{3}, x_{2}\right]=x_{5}, \alpha \in \mathbb{C} /\{-1,0\}, \beta \in \mathbb{C} /\{0\}, \alpha \beta \neq-1$, $(\alpha+1) \beta \neq-1$

L66: $\left[x_{1}, x_{1}\right]=x_{4},\left[x_{1}, x_{2}\right]=x_{4},\left[x_{2}, x_{1}\right]=x_{4}+x_{5},\left[x_{1}, x_{3}\right]=-x_{5}$, $\left[x_{2}, x_{3}\right]=-x_{5},\left[x_{3}, x_{2}\right]=x_{5}$

L80: $\left[x_{1}, x_{2}\right]=x_{4},\left[x_{2}, x_{1}\right]=x_{5},\left[x_{2}, x_{3}\right]=x_{4}$.

L82: $\left[x_{1}, x_{2}\right]=x_{4},\left[x_{2}, x_{1}\right]=-x_{4},\left[x_{2}, x_{3}\right]=x_{4},\left[x_{3}, x_{2}\right]=x_{5}$.

L83: $\left[x_{1}, x_{2}\right]=x_{4}+2 x_{5},\left[x_{2}, x_{1}\right]=-x_{5},\left[x_{2}, x_{2}\right]=x_{5},\left[x_{2}, x_{3}\right]=x_{4}$, $\left[x_{3}, x_{2}\right]=x_{5}$.

L89: $\left[x_{1}, x_{2}\right]=x_{4},\left[x_{2}, x_{1}\right]=-x_{4}+x_{5},\left[x_{1}, x_{3}\right]=-x_{5},\left[x_{3}, x_{1}\right]=x_{5}$, $\left[x_{2}, x_{3}\right]=x_{4}$.

$\mathrm{L} 89^{\prime}:\left[x_{1}, x_{2}\right]=x_{4},\left[x_{2}, x_{1}\right]=-x_{4}+x_{5},\left[x_{1}, x_{3}\right]=\gamma x_{5},\left[x_{3}, x_{1}\right]=x_{5}$, $\left[x_{2}, x_{3}\right]=x_{4}, \gamma \in \mathbb{C} /\{-1\}$.

$\mathrm{L} 89^{\prime \prime}:\left[x_{1}, x_{2}\right]=x_{4}+\alpha x_{5},\left[x_{2}, x_{1}\right]=\beta x_{4}+x_{5},\left[x_{1}, x_{3}\right]=-x_{5},\left[x_{3}, x_{1}\right]=$ $x_{5},\left[x_{2}, x_{3}\right]=x_{4}, \alpha \in \mathbb{C} /\{-1\}, \beta \in \mathbb{C}$.

L92: $\left[x_{1}, x_{2}\right]=x_{4},\left[x_{2}, x_{1}\right]=-x_{4},\left[x_{2}, x_{2}\right]=x_{5},\left[x_{1}, x_{3}\right]=-x_{5},\left[x_{3}, x_{1}\right]=$ $x 5,\left[x_{2}, x_{3}\right]=x_{4}$.

L92': $\left[x_{1}, x_{2}\right]=x_{4}+\alpha x_{5},\left[x_{2}, x_{1}\right]=\beta x_{4},\left[x_{2}, x_{2}\right]=x_{5},\left[x_{1}, x_{3}\right]=-x_{5}$, $\left[x_{3}, x_{1}\right]=x_{5},\left[x_{2}, x_{3}\right]=x_{4}, \alpha, \beta \in \mathbb{C}$ with $(\alpha, \beta) \neq(0,-1)$.

L94: $\left[x_{1}, x_{2}\right]=x_{4}-2 \beta x_{5},\left[x_{2}, x_{1}\right]=\alpha x_{4}+\beta x_{5},\left[x_{2}, x_{2}\right]=x_{5},\left[x_{1}, x_{3}\right]=$ $-x_{5},\left[x_{3}, x_{1}\right]=x_{5},\left[x_{2}, x_{3}\right]=x_{4}, \alpha \in \mathbb{C}, \beta \in \mathbb{C} /\{-1,0,1\}$.

L95: $\left[x_{1}, x_{2}\right]=x_{4}+\alpha x_{5},\left[x_{2}, x_{1}\right]=\beta x_{5},\left[x_{2}, x_{2}\right]=x_{5},\left[x_{1}, x_{3}\right]=-x_{5}$, $\left[x_{3}, x_{1}\right]=x_{5},\left[x_{2}, x_{3}\right]=x_{4}, \alpha \in \mathbb{C}, \beta \in \mathbb{C} /\{0\}, \alpha \neq-2 \beta, \beta^{2}+\alpha \beta+$ $1 \neq 0$.

$\mathrm{M1}:\left[x_{1}, x_{2}\right]=x_{4}-x_{5},\left[x_{2}, x_{1}\right]=-x_{4}+x_{5},\left[x_{1}, x_{3}\right]=\gamma x_{5},\left[x_{3}, x_{1}\right]=$ $-\gamma x_{5},\left[x_{2}, x_{3}\right]=x_{4},\left[x_{3}, x_{3}\right]=x_{5}, \gamma \in \mathbb{C}$.

$\mathrm{M1}^{\prime}:\left[x_{1}, x_{2}\right]=x_{4}-x_{5},\left[x_{2}, x_{1}\right]=-x_{4}+x_{5},\left[x_{1}, x_{3}\right]=\gamma x_{5},\left[x_{3}, x_{1}\right]=$ $\theta x_{5},\left[x_{2}, x_{3}\right]=x_{4},\left[x_{3}, x_{3}\right]=x_{5}, \gamma, \theta \in \mathbb{C}, \gamma \neq-\theta$.

$\mathrm{M} 2:\left[x_{1}, x_{2}\right]=x_{4},\left[x_{2}, x_{1}\right]=-x_{4},\left[x_{1}, x_{3}\right]=\theta x_{5},\left[x_{3}, x_{1}\right]=-\theta x_{5}$, $\left[x_{2}, x_{3}\right]=x_{4},\left[x_{3}, x_{3}\right]=x_{5}, \theta \in \mathbb{C}$.

$\mathrm{M}^{\prime}:\left[x_{1}, x_{2}\right]=x_{4},\left[x_{2}, x_{1}\right]=-x_{4},\left[x_{1}, x_{3}\right]=\theta x_{5},\left[x_{3}, x_{1}\right]=\delta x_{5},\left[x_{2}, x_{3}\right]=$ $x_{4},\left[x_{3}, x_{3}\right]=x_{5}, \delta, \theta \in \mathbb{C}, \delta \neq-\theta$.

$\mathrm{M}^{\prime \prime}:\left[x_{1}, x_{2}\right]=x_{4}+x_{5},\left[x_{2}, x_{1}\right]=-x_{4}-x_{5},\left[x_{1}, x_{3}\right]=\theta x_{5},\left[x_{3}, x_{1}\right]=$ $-\theta x_{5},\left[x_{2}, x_{3}\right]=x_{4},\left[x_{3}, x_{3}\right]=x_{5}, \theta \in \mathbb{C}$. 
$\mathrm{M} 2^{\prime \prime \prime}:\left[x_{1}, x_{2}\right]=x_{4}+x_{5},\left[x_{2}, x_{1}\right]=-x_{4}-x_{5},\left[x_{1}, x_{3}\right]=\theta x_{5},\left[x_{3}, x_{1}\right]=$ $\delta x_{5},\left[x_{2}, x_{3}\right]=x_{4},\left[x_{3}, x_{3}\right]=x_{5}, \gamma, \theta \in \mathbb{C}, \delta \neq-\theta$.

M7: $\left[x_{1}, x_{1}\right]=x_{4}+\alpha x_{5},\left[x_{1}, x_{2}\right]=\gamma x_{5},\left[x_{2}, x_{1}\right]=-\gamma x_{5},\left[x_{1}, x_{3}\right]=$ $\lambda x_{5},\left[x_{2}, x_{3}\right]=x_{4},\left[x_{3}, x_{2}\right]=-x_{4}, \alpha, \gamma, \lambda \in \mathbb{C}$.

M7': $\left[x_{1}, x_{1}\right]=x_{4}+\alpha x_{5},\left[x_{1}, x_{2}\right]=\beta x_{4}+\gamma x_{5},\left[x_{2}, x_{1}\right]=\theta x_{5},\left[x_{1}, x_{3}\right]=$ $\lambda x_{5},\left[x_{2}, x_{3}\right]=x_{4},\left[x_{3}, x_{2}\right]=-x_{4}, \alpha, \beta, \gamma, \lambda \in \mathbb{C}, \beta \neq 0$ or $\gamma \neq-\theta$.

M9: $\left[x_{1}, x_{1}\right]=x_{4}+\alpha x_{5},\left[x_{1}, x_{2}\right]=\beta x_{4}+\gamma x_{5},\left[x_{3}, x_{1}\right]=x_{5},\left[x_{1}, x_{3}\right]=$ $-x_{5},\left[x_{2}, x_{3}\right]=x_{4},\left[x_{3}, x_{2}\right]=-x_{4}, \alpha, \beta, \gamma \in \mathbb{C}, \beta \neq 0$ or $\gamma \neq 0$.

M9': $\left[x_{1}, x_{1}\right]=x_{4}+\alpha x_{5},\left[x_{1}, x_{2}\right]=\beta x_{4}+\gamma x_{5},\left[x_{3}, x_{1}\right]=x_{5},\left[x_{1}, x_{3}\right]=$ $\delta x_{5},\left[x_{2}, x_{3}\right]=x_{4},\left[x_{3}, x_{2}\right]=-x_{4}, \alpha, \beta, \gamma \in \mathbb{C}, \delta \neq-1, \beta \neq 0$ or $\gamma \neq 0$.

M11: $\left[x_{1}, x_{1}\right]=x_{4}+\alpha x_{5},\left[x_{1}, x_{2}\right]=\beta x_{4}+\gamma x_{5},\left[x_{2}, x_{1}\right]=\theta x_{5},\left[x_{2}, x_{3}\right]=$ $-x_{4},\left[x_{3}, x_{2}\right]=x_{4}, \alpha, \beta, \gamma, \theta \in \mathbb{C}, \beta \neq 0$ or $\gamma \neq-\theta$.

M12: $\left[x_{1}, x_{1}\right]=x_{4},\left[x_{1}, x_{2}\right]=\alpha x_{4}+\beta x_{5},\left[x_{2}, x_{1}\right]=\gamma x_{5},\left[x_{1}, x_{3}\right]=x_{5}$, $\left[x_{2}, x_{3}\right]=-x_{4},\left[x_{3}, x_{2}\right]=x_{4}, \alpha, \beta, \gamma, \theta \in \mathbb{C}, \alpha \neq 0$ or $\gamma \neq-\beta$.

M13: $\left[x_{1}, x_{1}\right]=x_{4}+\alpha x_{5},\left[x_{1}, x_{2}\right]=\gamma x_{5},\left[x_{2}, x_{1}\right]=-\gamma x_{5},\left[x_{3}, x_{1}\right]=$ $x_{5},\left[x_{1}, x_{3}\right]=\lambda x_{5},\left[x_{2}, x_{3}\right]=-x_{4},\left[x_{3}, x_{2}\right]=x_{4}, \alpha, \gamma, \lambda \in \mathbb{C}, \lambda \neq-1$.

M13': $\left[x_{1}, x_{1}\right]=x_{4}+\alpha x_{5},\left[x_{1}, x_{2}\right]=\beta x_{4}+\gamma x_{5},\left[x_{2}, x_{1}\right]=\theta x_{5},\left[x_{3}, x_{1}\right]=$ $x_{5},\left[x_{1}, x_{3}\right]=\lambda x_{5},\left[x_{2}, x_{3}\right]=-x_{4},\left[x_{3}, x_{2}\right]=x_{4}, \alpha, \beta, \gamma, \theta, \lambda \in \mathbb{C}$, $\lambda \neq-1$, and $\beta \neq 0$ or $\gamma \neq-\theta$.

M15: $\left[x_{1}, x_{2}\right]=-x_{4}+\gamma x_{5},\left[x_{2}, x_{1}\right]=x_{4}-\gamma x_{5},\left[x_{3}, x_{1}\right]=\mu x_{5},\left[x_{1}, x_{3}\right]=$ $x_{4}+\lambda x_{5},\left[x_{2}, x_{3}\right]=\omega x_{5},\left[x_{3}, x_{2}\right]=x_{4}, \gamma, \mu, \lambda \omega \in \mathbb{C}$.

Proof. The result is obtained by checking each Leibniz algebra of the following theorems in [10]. Again, for the convenience of the reader, we kept the same numbering as in [10], and just differentiated theorems by the Letters as follows: Theorem 4.2.3 for the Bs, Theorem 4.2.5 for the Cs, Theorem 4.2.6 for the Ds, Theorem 4.2.7 for the Es, Theorem 4.2.9 for the Fs, Theorem 4.2.10 for the Gs, Theorem 4.2.11 for the Hs, Theorem 4.3.1 for the Is, Theorem 4.3.2 for the Js, Theorem 4.3.3 for the Ks, Theorem 4.3.4 for the Ls and Ms.

The basis of the corresponding Lie-centers and absolute maximal Lie subalgebras are given in the table below:

\begin{tabular}{|l|l|l|}
\hline \multicolumn{2}{|c|}{ 5-dimensional non-split nilpotent s-Leibniz algebras } \\
\hline s-Leibniz algebra $\mathfrak{g}$ & Lie-center & Maximal Lie \\
& $Z_{\text {Lie }}(\mathfrak{g})$ & subalgebra $\mathfrak{s}$ \\
\hline \hline$B 1, B 3$ & $\left\{x_{3}, x_{4}, x_{5}\right\}$ & $\left\{x_{1}, x_{3}, x_{4}, x_{5}\right\}$ \\
$C 2, C 4$ & $\left\{x_{3}, x_{4}, x_{5}\right\}$ & $\left\{x_{1}, x_{3}, x_{4}, x_{5}\right\}$ \\
$C 8$ & $\left\{x_{3}, x_{4}, x_{5}\right\}$ & $\left\{x_{2}, x_{3}, x_{4}, x_{5}\right\}$ \\
\hline
\end{tabular}




\begin{tabular}{|c|c|c|}
\hline \multicolumn{3}{|c|}{ 5-dimensional non-split nilpotent s-Leibniz algebras } \\
\hline s-Leibniz algebra $\mathfrak{g}$ & $\begin{array}{l}\text { Lie-center } \\
Z_{\text {Lie }}(\mathfrak{g})\end{array}$ & $\begin{array}{l}\text { Maximal Lie } \\
\text { subalgebra } \mathfrak{s}\end{array}$ \\
\hline $\begin{array}{l}D 1, D 8, D 14, D 15, D 18 \\
E 1 \\
E 10 \\
F 1\end{array}$ & $\begin{array}{l}\left\{x_{3}, x_{4}, x_{5}\right\} \\
\left\{x_{4}, x_{5}\right\} \\
\left\{x_{2}, x_{3}, x_{5}\right\} \\
\left\{x_{3}, x_{4}, x_{5}\right\}\end{array}$ & $\begin{array}{l}\left\{x_{2}, x_{3}, x_{4}, x_{5}\right\} \\
\left\{x_{2}, x_{3}, x_{4}, x_{5}\right\} \\
\left\{x_{2}, x_{3}, x_{4}, x_{5}\right\} \\
\left\{x_{1}, x_{3}, x_{4}, x_{5}\right\}, \\
\left\{x_{2}, x_{3}, x_{4}, x_{5}\right\}\end{array}$ \\
\hline $\begin{array}{l}F 3 \\
G 1 \\
G 3 \\
H 1\end{array}$ & $\begin{array}{l}\left\{x_{3}, x_{4}, x_{5}\right\} \\
\left\{x_{3}, x_{4}, x_{5}\right\} \\
\left\{x_{3}, x_{4}, x_{5}\right\} \\
\left\{x_{3}, x_{4}, x_{5}\right\}\end{array}$ & $\begin{array}{l}\left\{x_{1}, x_{3}, x_{4}, x_{5}\right\} \\
\left\{x_{1}, x_{3}, x_{4}, x_{5}\right\} \\
\left\{x_{2}, x_{3}, x_{4}, x_{5}\right\} \\
\left\{x_{1}, x_{3}, x_{4}, x_{5}\right\}, \\
\left\{x_{2}, x_{3}, x_{4}, x_{5}\right\}\end{array}$ \\
\hline $\begin{array}{l}H 3, H 5 \\
I 6\end{array}$ & $\begin{array}{l}\left\{x_{3}, x_{4}, x_{5}\right\} \\
\left\{x_{2}, x_{4}, x_{5}\right\}\end{array}$ & $\begin{array}{l}\left\{x_{1}, x_{3}, x_{4}, x_{5}\right\} \\
\left\{x_{1}, x_{2}, x_{4}, x_{5}\right\}, \\
\left\{x_{2}, x_{3}, x_{4}, x_{5}\right\}\end{array}$ \\
\hline$I 8, I 14$ & $\left\{x_{1}, x_{4}, x_{5}\right\}$ & $\begin{array}{l}\left\{x_{1}, x_{2}, x_{4}, x_{5}\right\}, \\
\left\{x_{1}, x_{3}, x_{4}, x_{5}\right\}\end{array}$ \\
\hline$I 13$ & $\left\{x_{3}, x_{4}, x_{5}\right\}$ & $\begin{array}{l}\left\{x_{1}, x_{3}, x_{4}, x_{5}\right\}, \\
\left\{x_{2}, x_{3}, x_{4}, x_{5}\right\}\end{array}$ \\
\hline $\begin{array}{l}I 18 \\
J 8 \\
J 10 \\
K 2 \\
K 4\end{array}$ & $\begin{array}{l}\left\{x_{3}, x_{4}, x_{5}\right\} \\
\left\{x_{2}, x_{3}, x_{5}\right\} \\
\left\{x_{3}, x_{5}\right\} \\
\left\{x_{2}, x_{4}, x_{5}\right\} \\
\left\{x_{2}, x_{4}, x_{5}\right\}\end{array}$ & $\begin{array}{l}\left\{x_{1}, x_{3}, x_{4}, x_{5}\right\} \\
\left\{x_{2}, x_{3}, x_{4}, x_{5}\right\} \\
\left\{x_{2}, x_{3}, x_{4}, x_{5}\right\} \\
\left\{x_{2}, x_{3}, x_{4}, x_{5}\right\} \\
\left\{x_{1}, x_{2}, x_{4}, x_{5}\right\}, \\
\left\{x_{2}, x_{3}, x_{4}, x_{5}\right\}\end{array}$ \\
\hline $\begin{array}{l}K 5 \\
K 6 \\
K 9 \\
K 12 \\
L 14, L 16, L 35, L 44, L 46, \\
L 50, L 80, L 83, L 89^{\prime \prime}, L 92^{\prime}, \\
L 94, L 95\end{array}$ & $\begin{array}{l}\left\{x_{3}, x_{4}, x_{5}\right\} \\
\left\{x_{2}, x_{4}, x_{5}\right\} \\
\left\{x_{4}, x_{5}\right\} \\
\left\{x_{3}, x_{4}, x_{5}\right\} \\
\left\{x_{4}, x_{5}\right\}\end{array}$ & $\begin{array}{l}\left\{x_{2}, x_{3}, x_{4}, x_{5}\right\} \\
\left\{x_{1}, x_{2}, x_{4}, x_{5}\right\} \\
\left\{x_{1}, x_{2}, x_{4}, x_{5}\right\} \\
\left\{x_{1}, x_{3}, x_{4}, x_{5}\right\}, \\
\left\{x_{2}, x_{3}, x_{4}, x_{5}\right\} \\
\left\{x_{1}, x_{3}, x_{4}, x_{5}\right\}\end{array}$ \\
\hline $\begin{array}{l}L 36, L 89, L 92 \\
L 42, L 55, L 60, L 61, L 63, L 66 \\
L 58 \\
L 45, L 56\end{array}$ & $\begin{array}{l}\left\{x_{1}, x_{4}, x_{5}\right\} \\
\left\{x_{4}, x_{5}\right\} \\
\left\{x_{2}, x_{4}, x_{5}\right\} \\
\left\{x_{3}, x_{4}, x_{5}\right\}\end{array}$ & $\begin{array}{l}\left\{x_{1}, x_{3}, x_{4}, x_{5}\right\} \\
\left\{x_{2}, x_{3}, x_{4}, x_{5}\right\} \\
\left\{x_{2}, x_{3}, x_{4}, x_{5}\right\} \\
\left\{x_{2}, x_{3}, x_{4}, x_{5}\right\}\end{array}$ \\
\hline
\end{tabular}




\begin{tabular}{|l|l|l|}
\hline \multicolumn{3}{|c|}{ 5-dimensional non-split nilpotent s-Leibniz algebras } \\
\hline s-Leibniz algebra $\mathfrak{g}$ & Lie-center & Maximal Lie \\
& $Z_{\text {Lie }}(\mathfrak{g})$ & subalgebra $\mathfrak{s}$ \\
\hline \hline$L 82, L 89^{\prime}, L 92$ & $\left\{x_{1}, x_{4}, x_{5}\right\}$ & $\left\{x_{1}, x_{2}, x_{4}, x_{5}\right\}$ \\
$M 1, M 2, M 2^{\prime \prime}$ & $\left\{x_{1}, x_{4}, x_{5}\right\}$ & $\left\{x_{1}, x_{2}, x_{4}, x_{5}\right\}$ \\
$M 1^{\prime}, M 2^{\prime}, M 2^{\prime \prime \prime}, M 15$ & $\left\{x_{4}, x_{5}\right\}$ & $\left\{x_{1}, x_{2}, x_{4}, x_{5}\right\}$ \\
$M 7, M 13$ & $\left\{x_{2}, x_{4}, x_{5}\right\}$ & $\left\{x_{2}, x_{3}, x_{4}, x_{5}\right\}$ \\
$M 8, M 10, M 12, M 14$ & $\left\{x_{4}, x_{5}\right\}$ & $\left\{x_{2}, x_{3}, x_{4}, x_{5}\right\}$ \\
$M 9, M 11$ & $\left\{x_{3}, x_{4}, x_{5}\right\}$ & $\left\{x_{2}, x_{3}, x_{4}, x_{5}\right\}$ \\
\hline
\end{tabular}

\section{References}

[1] R.K. Amayo: Quasi-ideals of Lie algebras II, Proc. Lond. Math. Soc. , 3 (33), (1976), 37-64.

[2] Sh. A. Ayupov and B. A. Omirov: On some classes of nilpotent Leibniz algebras, Sibirsk. Mat. Zh., 42 (1), (2001), 18-29.

[3] G. R. Biyogmam and J. M. Casas: On Lie-isoclinic Leibniz algebras, J. Algebra 499 (2018), 337-357.

[4] G. R. Biyogmam and J. M. Casas: The c-Nilpotent Shur Lie-Multiplier of Leibniz Algebras, J. Geom. Phys. 138 (2019), 55-69.

[5] D. Barnes: On Levi's theorem for Leibniz algebras, Bull. Aust. Math. Soc. 86 (2012), 184-185

[6] J. M. Casas and E. Khmaladze: On Lie-central extensions of Leibniz algebras, RACSAM (2016), DOI 10.1007/s13398-016-0274-6.

[7] J. M. Casas and T. Van der Linden: A relative theory of universal central extensions, Pré-Publicaçoes do Departamento de Matemàtica, Universidade de Coimbra Preprint Number 09- (2009).

[8] J. M. Casas and M. A. Insua: The Schur Lie-multiplier of Leibniz algebras, Quaestiones Mathematicae, 41 (2) (2018).

[9] J. M. Casas and T. Van der Linden: Universal central extensions in semi-abelian categories, Appl. Categor. Struct. 22 (1) (2014), 253-268.

[10] I. Demir, Classification of 5-Dimensional Complex Nilpotent Leibniz Algebras., Ph.D. Thesis, http://www.lib.ncsu.edu/resolver/1840.20/33418, 138 pages.

[11] I. Demir, C. Kailash and E. Stitzinger: On classification of four-dimensional nilpotent Leibniz algebras, Comm. Algebra 45 (3) (2017), 1012-1018.

[12] I. Demir, C. Kailash and E. Stitzinger: On some structure of Leibniz algebras, in Recent Advances in Representation Theory, Quantum Groups, Algebraic Geometry, and Related Topics, Contemporary Mathematics, 623, Amer. Math. Soc., Providence, RI, 41-54 (2014).

[13] V. Gorbatsevich: On some structure of Leibniz algebras, arxiv:1302.3345v2.

[14] G. Janelidze, L. Màrki and W. Tholen: Semi-abelian categories, J. Pure Appl. Algebra 168 (2002), 367-386. 
[15] J.-L. Loday: Cyclic homology, Grundl. Math. Wiss. Bd. 301, Springer (1992).

[16] J.-L. Loday: Une version non commutative des algèbres de Lie: les algèbres de Leibniz, L'Enseignement Mathématique 39 (1993), 269-292.

[17] D. Towers: Lie algebras all of whose maximal subalgebras have codimension one, Proc. Edin. Math. Soc. 24 (1981), 217-219.

[18] D. Towers: Maximal subalgebras of Lie algebras containing Engel subalgebras, J. Pure Appl. Algebra 216 (2012), 688-693.

\section{CONTACT INFORMATION}

G. R. Biyogmam Department of Mathematics, Georgia College \& State University, Campus Box 17 Milledgeville, GA 31061-0490 E-Mail(s): guy.biyogmam@gcsu .edu

Calvin Tcheka Department of Mathematics, University of Dschang, Dschang, Cameroun E-Mail(s): jtcheka@gmail.com

Received by the editors: 15.05.2018. 